\title{
Packet Efficient Implementation of the Omega Failure Detector
}

\author{
Quentin Bramas $^{1}$, Dianne Foreback ${ }^{2}$, Mikhail Nesterenko ${ }^{2}$, and Sébastien Tixeuil ${ }^{1,3}$ \\ 1 Sorbonne Universités, UPMC Univ Paris 06, CNRS, LIP6 UMR 7606, 4 place Jussieu 75005 Paris \\ 2 Kent State University \\ 3 Institut Universitaire de France
}

\begin{abstract}
We assume that a message may be delivered by packets through multiple hops and investigate the feasibility and efficiency of an implementation of the Omega Failure Detector under such an assumption. To motivate the study, we prove that the existence and sustainability of a leader is exponentially more probable in a multi-hop Omega implementation than in a single-hop one.

An implementation is: message efficient if all but finitely many messages are sent by a single process; packet efficient if the number of packets used to transmit a message in all but finitely many messages is linear w.r.t the number of processes, packets of different messages may potentially use different channels, thus the number of used channels is not limited; super packet efficient if the number of channels used by packets to transmit all but finitely many messages is linear.

We present the following results for deterministic algorithms. If reliability and timeliness of one message does not correlate with another, i.e., there are no channel reliability properties, then a packet efficient implementation of Omega is impossible. If eventually timely and fair-lossy channels are considered, we establish necessary and sufficient conditions for the existence of a message and packet efficient implementation of Omega. We also prove that the eventuality of timeliness of channels makes a super packet efficient implementation of Omega impossible. On the constructive side, we present and prove correct a deterministic packet efficient implementation of Omega that matches the necessary conditions we established.
\end{abstract}

\section{Introduction}

The asynchronous system model places no assumptions on message propagation delay or relative process speeds. This makes the model attractive for distributed algorithm research as the results obtained in the model are applicable to an arbitrary network and computer architecture. However, the fully asynchronous system model is not well suited for fault tolerance studies. An elementary problem of consensus, where processes have to agree on a single value, is unsolvable even if only one process may crash [9]: the asynchrony of the model precludes processes from differentiating a crashed and a slow process.

A failure detector [6] is a construct that enables the solution to consensus or related problems in the asynchronous system model. Potentially, a failure detector may be very powerful and, therefore, hide the solution to the problem within its specification. Conversely, the weakest failure detector specifies the least amount of synchrony required to implement consensus [5]. One such detector is Omega ${ }^{4}$,

Naturally, a failure detector may not be implemented in the asynchronous model itself. Hence, a lot of research is focused on providing the implementation of a detector, especially Omega, in the least restrictive communication model. These restrictions deal with

\footnotetext{
${ }^{4}$ In literature, the detector is usually denoted by the Greek letter. However, we use the letter to denote low complexity bound. To avoid confusion, we spell out the name of the failure detector in English.
} 
timeliness and reliability of message delivery. Aguilera et al. [1] provide a remarkable Omega implementation which requires only a single process to have eventually timely channels to the other processes and a single process to have so called fair-lossy channels to and from all other processes. Aguilera et al. present what they call an efficient implementation where only a single process sends infinitely many messages. In their work, Aguilera et al. consider a direct channel as the sole means of message delivery from one process to another. In this paper, we consider a more general setting where a message may arrive to a particular process through several intermediate processes. Otherwise, we preserve model assumptions of Aguilera et al.

Our contribution. We study Omega implementation under the assumption that a message may come to its destination through other processes.

To motivate this multi-hop Omega implementation approach, we consider a fixed probability of channel timeliness and study the probability of leader existence in a classic single-hop and in multi-hop implementations. We prove that the probability of leader existence tends to zero for single-hop implementations and to one for multi-hop ones as network size grows. Moreover, probability of leader persisting while the timeliness of channel changes tends to zero for single-hop and to infinity for multi-hop implementations.

If we consider deterministic algorithms, we study three classes of Omega implementations: message efficient, packet efficient and super packet efficient. In a message efficient implementation all but finitely many messages are sent by a single process. In a packet efficient implementation, the number of packets in all but finitely many transmitted messages is linear w.r.t. the number of processes in the network. However, in a (simple) packet efficient implementation, packets of different messages may use different channels such that potentially all channels in the system are periodically used. In a super packet efficient implementation, the number of channels used in all but finitely many messages is also linear w.r.t. to the number of processes.

Our major results are as follows. If timeliness of one message does not correlate with the timeliness of another, i.e., there are no timely channels, we prove that any implementation of Omega has to send infinitely many messages whose number of packets is quadratic w.r.t to the number of processes in the network. This precludes a packet efficient implementation of Omega. If eventually timely and fair-lossy channels are allowed, we establish the necessary and sufficient conditions for the existence of a packet efficient implementation of Omega. We then prove that this eventuality of timely and channels precludes the existence of a super packet efficient implementation of Omega. We present an algorithm that uses these necessary conditions provides a message and packet efficient implementation of Omega

Related work. The implementation of failure detectors is a well-researched area [2/3 $8|11| 12|13| 14|15| 16 \mid 17]$ Refer to [12] for detailed comparisons of work related to the kind of Omega implementation we are proposing. We are limiting our literature review to the most recent and closest to ours studies. 
Delporte-Gallet et al. [8] describe algorithms for recognizing timely channel graphs. Their algorithms are super packet efficient and may potentially be used to implement Omega. However, their solutions assume non-constant size messages and perpetually reliable channels. That is Delporte-Gallet et al. deviate from the model of Aguilera et al. and the algorithms of Delporte-Gallet et al. do not operate correctly under fair-lossy and eventually timely channel assumptions.

A number of papers consider Omega implementation under various modifications of Aguilera et al model. Hutle et al. [11] implement Omega assuming a send-to-all message transmission primitive where $f$ processes are guaranteed to receive the message timely. Fernandez and Raynal [2] assume a process that is able to timely deliver its message to a quorum of processes over direct channels. This quorum and channels may change with each message. A similar rotating set of timely channels is used by Malkhi et al. [14]. Larrea et al. [13] give an efficient implementation of Omega but assume that all channels are eventually timely. In their Omega implementation, Mostefaoui et al. [15] rely on a particular order of message interleaving rather than on timeliness of messages. Biely and Widder [3] consider message-driven (i.e., non-timer based) model and provide an efficient Omega implementation.

There are several recent papers on timely solutions to problems related to Omega implementation. Charron-Bost et al. [7] use a timely spanning tree to solve approximate consensus. Lafuente et al. [12] implement eventually perfect failure detector using a timely cycle of processes.

\section{Notation and Definitions}

Model specifics. To simplify the presentation, we use an even more general model than what is used in Aguilera et al. [1]. The major differences are as follows. We use infinite capacity non-FIFO channels rather than single packet capacity channels. Our channel construct makes us explicitly state the packet fairness propagation assumptions that are somewhat obscured by the single capacity channels.

In addition, we do not differentiate between a slow process and a slow channel since slow channels may simulate both. Omega implementation code is expressed in terms of guarded commands, rather than the usual more procedural description. The operation of the algorithm is a computation which is a sequence of these command executions. We express timeouts directly in terms of computation steps rather than abstract or concrete time. This simplifies reasoning about them.

Despite the differences, the models are close enough such that all of the results in this paper are immediately applicable to the traditional Omega implementation model.

Processes and computations. A computer network consists of a set $N$ of processes. The cardinality of this set is $n$. Each process has a unique identifier from 0 to $n-1$. Processes interact by passing messages through non-FIFO unbounded communication channels. Each process has a channel to all other processes. That is, the network is fully connected. A message is constant size if the data it carries is in $O(\log n)$. For example, a constant size message may carry several process identifiers but not a complete network spanning tree. 
Each process has variables and actions. The action has a guard: a predicate over the local variables and incoming channels of the process. An action is enabled if its guard evaluates to true. A computation is a potentially infinite sequence of global network states such that each subsequent state is obtained by executing an action enabled in the previous state. This execution is a computation step. Processes may crash. Crashed process stops executing its actions. Correct process does not crash.

Messages and packets. We distinguish between a packet and a message. Message is particular content to be distributed to processes in the network. Origin is the process that initiates the message. The identifier of the origin is included in the message. Messages are sent via packets. Packet is a portion of data transmitted over a particular channel. A message is the payload of a packet. A process may receive a packet and either forward the message it contains or not. A process may not modify it: if a process needs to send additional information, the process may send a separate message. A process may forward the same message at most once. In effect, a message is transmitted to processes of the network using packets. A particular process may receive a message either directly from the origin, or indirectly possibly through multiple hops.

Scheduling and fairness. We express process synchronization in terms of an adversarial scheduler. The scheduler restrictions are as follows. We do not distinguish slow processes and slow packet propagation. A scheduler may express these phenomena through scheduling process action execution in a particular way. A packet transmission immediately enables the packet receipt action in the recipient process. A packet is lost if the receipt action is never executed. A packet is not lost if it is eventually received.

Timers. Timer is a construct with the following properties. A timer can be reset, stopped and increased. It can also be checked whether the timer is on or off. It has a timeout integer value and a timeout action associated with it. A timer is either a receiver timer or a sender timer. If a sender timer is on, timeout action is executed once the computation has at most the timeout integer steps without executing the timer reset. If a receiver timer is on, the timeout action is executed once the computation has at least the timeout integer steps without executing the timer reset. Increasing the timer, adds an arbitrary positive integer value to the timeout integer. An off timer can be set to on by resetting it.

Reliable and timely messages and packets. A packet is reliable if it is received. A message is reliable if it is received by every correct process. A channel is reliable if every packet transmitted over this channel is reliable.

A channel is fair-lossy if it has the following properties. If there is an infinite number of packet transmissions over a particular fair-lossy channel of a particular message type and origin, then infinitely many are received. We assume that a fair-lossy channel is not type discriminating. That is, if it is fair-lossy for one type and origin, it is also fair-lossy for every pair of message type and origin.

Observe that if there is an infinite number of message transmissions of a particular message type and origin over a path that is fair-lossy, then infinitely many succeed. There 
converse is true as well: if there is an infinite number of successful message transmissions, there must be a fair-lossy path between the origin an the destination.

A packet is timely if it is received within a bounded number of computation steps. Specifically, there is a finite integer $B$ such that the packet is received within $B$ steps. Naturally, a timely packet is a reliable packet. A message is timely if it is received by every process via a path of timely packets. A channel is timely if every packet transmitted over this channel is timely. A channel is eventually timely if the number of non-timely packets it transmits is finite. Note that a channel that transmits a finite number of packets is always eventually timely.

The timely channel definition is relatively clear. The opposite, non-timely channel, is a bit more involved. A channel that occasionally delays or misses a few packets is not nontimely as the algorithm may just ignore the missed packets with a large enough timeout. Hence, the following definition.

A channel is strongly non-timely if the following holds. If there is an infinite number of packet transmissions of a particular type and origin over a particular non-timely channel, then, for any fixed integer, there are infinitely many computation segments of this length such that none of the packets are delivered inside any of the segments.

Similarly, the non-timeliness has to be preserved across multiple channels, a message may not gain timeliness by finding a parallel timely path, then, for example, the two paths may alternate delivering timely messages. Therefore, we add an additional condition for non-timeliness.

All paths between a pair of processes $x$ and $y$ are strongly non-timely if $x$ sends an infinite number of messages to $y$, yet regardless of how the message is forwarded or what path it takes, for any fixed integer, there are infinitely many computation segments of this length such that none of the messages are delivered inside any of the segments. Unless otherwise noted, when we discuss non-timely channels and paths, we mean strongly nontimely channels and paths.

Communication models. To make it easier to address the variety of possible communication restrictions, we define several models. The dependable (channel) model allows eventually or perpetually reliable timely or fair-lossy channels. In the dependable model, an algorithm may potentially discover the dependable channels by observing packet propagation. The general propagation model does not allow either reliable or timely channels. Thus, one message propagation is not related to another message propagation.

Message propagation graph. Message propagation graph is a directed graph over network processes and channels that determines whether packet propagation over a particular channel would be successful. This graph is connected and has a single source: the origin process. This concept is a way to reason about scheduling of the packets of a particular message.

Each message has two propagation graphs. In reliable propagation graph $R$, each edge indicates whether the packet is received if transmitted over this channel. In timely propagation graph $T$ each edge indicates whether the packet is timely if transmitted over this channel. Since a timely packet is a reliable packet, for the same message, the timely prop- 
agation graph is a subgraph of the reliable propagation graph. In general, a propagation graph for each message is unique. That is, even for the same source process, the graphs for two messages may differ. This indicates that different messages may take divergent routes.

If a channel from process $x$ to process $y$ is reliable, then edge $(x, y)$ is present in the reliable propagation graph for every message where process $x$ is present. In other words, if the message reaches $x$ and $x$ sends it to $y$, then $y$ receives it. A similar discussion applies to a timely channel and corresponding edges in timely propagation graphs.

Propagation graphs are determined by the scheduler in advance of the message transmission. That is, the recipient process, depending on the algorithm, may or may not forward the received message along a particular outgoing channel. However, if the process forwards the message, the presence of an edge in the propagation graph determines the success of the message transmission. Note that the process forwards a particular message at most once. Hence, the propagation graph captures the complete possible message propagation pattern. A process may crash during message transmission. This crash does not alter propagation graphs.

Proposition 1. A message is reliable only if its reliable propagation graph $R$ is such that every correct process is reachable from the origin through non-crashed processes.

Proposition 2. A message is timely only if its timely propagation graph $T$ is such that every correct process is reachable from the origin through non-crashed processes.

Omega implementation and its efficiency. An algorithm that implements the Omega Failure Detector (or just Omega) is such that in a suffix of every computation, each correct process outputs the identifier of the same correct process. This process is the leader.

An implementation of Omega is message efficient if the origin of all but finitely many messages is a single correct process and all but finitely many messages are constant size. An implementation of Omega is packet efficient if all but finitely many messages are transmitted using $O(n)$ packets.

An omega implementation is super packet efficient if it is packet efficient and the packets of all but finitely many messages are using the same channels. In other words, if a packet of message $m_{1}$ is forwarded over some channel, then a packet of another message $m_{2}$ is also forwarded over this channel. The intent of a super packet efficient algorithm is to only use a limited number of channels infinitely often. Since a packet efficient algorithm uses $O(n)$ packets infinitely often, a super packet efficient algorithm uses $O(n)$ channels infinitely often.

\section{Probabilistic Properties}

In this section, we contrast a multi-hop implementation of Omega and a classic singlehop, also called direct channel, implementation. We assume each network channel is timely with probability $p$. The timeliness probability of one channel is independent of this probability of any other channel. 
Leader existence probability. We assume that the leader may exist only if there is a process that has timely paths to all processes in the network. In case of direct channel implementation, the length of each such path must be exactly one.

As $n$ grows, Omega implementations behave radically differently. Theorems 1 and 2 state the necessary conditions for leader existence and indicate that the probability of leader existence for direct channel implementation approaches zero exponentially quickly, while this probability for multi-hop implementation approaches one exponentially quickly. In practical terms, a multi-hop omega implementation is far more likely to succeed in establishing the leader.

Theorem 1. If the probability of each channel to be timely is $p<1$, then the probability of leader existence in any direct channel Omega implementation approaches zero exponentially fast as $n$ grows.

Proof: Let $D_{x}$ be the probability that some process $x$ does not have direct timely channels to all processes in the network. This probability is $\mathbb{P}\left(D_{x}\right)=1-p^{n-1}$. For two distinct processes $x$ and $y, D_{x}$ and $D_{y}$ are disjoint since channels are oriented. Thus, if $p<1$, the probability that no leader exists is $\mathbb{P}\left(\bigcap_{x \in V} D_{x}\right)=\left(1-p^{n-1}\right)^{n} \stackrel{n \rightarrow+\infty}{\rightarrow} 1$.

Theorem 2. If the probability of each channel to be timely is $p<1$, then the probability of leader existence in any multi-hop Omega implementation approaches 1 exponentially fast as $n$ grows.

Proof: A channel is bitimely if it is timely in both directions. The probability that there exists at least one process such that there exist timely paths from this process to all other processes is greater than the probability to reach them through bitimely paths. We use the probability of the latter as a lower bound for our result. If $p$ is the probability of a channel to be timely, $\tilde{p}=p^{2}$ is the probability that it is bitimely. Consider graph $G$ where the edges represent bitimely channels. It is an Erdos-Renyi graph where an edge exists with probability $\tilde{p}$. It was shown (see [10]) that $\mathbb{P}\left(G\right.$ is connected) $\sim 1-n(1-\tilde{p})^{n-1} \stackrel{n \rightarrow+\infty}{\rightarrow} 1$.

Leader stability. As in previous subsection, we assume the leader has timely paths to all other processes in the network. If channel timeliness changes, this process may not have timely paths to all other processes anymore. Leader stability time is the expected number of rounds of such channel timeliness change where a particular process remains the leader.

Again, direct channel and multi-hop implementations of Omega behave differently. Direct channel leader stability time approaches zero as $n$ increases and cannot be limited from below by fixing a particular value of channel timeliness probability. Multi-hop leader stability goes to infinity exponentially quickly. In a practical setting, a leader is significantly more stable in a multi-hop Omega implementation than in a direct channel one. 
Theorem 3. In any direct channel Omega implementation, if the probability of each channel to be timely is $p<1$, leader stability time goes exponentially fast to 0 as $n$ grows. If leader stability time is to remain above a fixed constant $E>0$, then the channel timeliness probability $p$ must converge to 1 exponentially fast as $n$ grows.

Proof: At a given time, a given process has timely channels to all other processes with probability $p^{n-1}$. The number of rounds $X$ a given process retains timely paths to all other processes follows a geometric distribution $\mathcal{P}(X=r)=q^{r}(1-q)$, where $q=p^{n-1}$. Thus, the expected number of rounds a process retains timely channels to all other processes is $\frac{q}{1-q}=\frac{p^{n-1}}{1-p^{n-1}} \sim p^{n-1}$, which tends exponentially fast toward 0 if $p$ is a constant less than 1 .

Assume $\mathbb{E}(X)$ converges towards a given fixed number $E$ as $n$ tends towards infinity. That is, we need $\lim _{n \rightarrow \infty} \mathbb{P}(G$ is connected $)=\frac{1}{E+1}$. Then, $p^{n-1}$ tends to $\frac{1}{E+2}$, which implies that $p$ converges towards 1 exponentially fast.

Theorem 4. In any multi-hop Omega implementation, if the probability of each channel to be timely is $p<1$, leader stability time goes to infinity exponentially fast as $n$ grows. If leader stability time is to remain above a fixed constant $E>0$, then channel timeliness probability may converge to 0 exponentially fast as $n$ grows.

Proof: If we fix $\tilde{p}, 0<\tilde{p}<1$, we have $\mathbb{P}(G$ is connected $) \sim 1-n(1-\tilde{p})^{n-1}$ (see [10]). Then, the expected number of rounds a given process retains timely paths to all other processes is asymptotically $n^{-1}\left(\frac{1}{1-\tilde{p}}\right)^{n}$, which increases exponentially fast.

Assume $\mathbb{E}(X)$ converges towards a given fixed number $E$ as $n$ tends to infinity. This means that

$$
\lim _{n \rightarrow \infty} \mathbb{P}(G \text { is connected })=\frac{1}{E+1}=e^{-e^{-c}}
$$

Using well-known results of random graph theory [4], we can take

$$
\tilde{p}(n)=\frac{\ln n}{n}+\frac{c}{n}=\frac{\ln n}{n}-\frac{\ln \ln (1+E)}{n}
$$

\section{Necessity and Sufficiency Properties}

We now explore the properties of deterministic Omega implementation.

Model independent properties. The below Omega implementation properties are applicable to both general propagation and dependable channel model.

Theorem 5. In an implementation of Omega, at least one correct process needs to send infinitely many timely messages.

Proof: Assume $\mathcal{A}$ is an implementation of Omega where every correct process sends a finite number of timely messages. Start with a network where all but two processes $x$ and $y$ crash, wait till all timely messages are sent. Since $\mathcal{A}$ is an implementation of Omega, 
eventually $x$ and $y$ need to agree on the leader. Let it be $x$. Since all timely messages are sent, the remaining messages may be delayed arbitrarily. If $x$ now crashes, process $y$ must eventually elect itself the leader. Instead, we delay messages from $x$ to $y$. The crash and the delay are indistinguishable to $y$ so it elects itself the leader. We now deliver messages in an arbitrary manner. Again, since $\mathcal{A}$ implements Omega, $x$ and $y$ should agree on the leader. Let it be $y$. The argument for $x$ is similar. We then delay messages from $y$ to $x$ forcing $x$ to select itself the leader. We continue this procedure indefinitely. The resultant sequence is a computation of $\mathcal{A}$. However at least one process, either $x$ or $y$, oscillates in its leader selection infinitely many times. To put another way, this process never elects the leader. This means that, contrary to the initial assumption, $\mathcal{A}$ is not an implementation of Omega. This proves the theorem.

If single process sends an infinite number of messages in a message efficient implementation of Omega, this process must be the leader. Otherwise processes are not able to recognize the crash of the leader. Hence, the corollary of Theorem 5 .

Corollary 1. In a message efficient implementation of Omega, the leader must send infinitely many timely messages.

\section{General propagation model properties.}

Lemma 1. To timely deliver a message in the general propagation model, each recipient process needs to send it across every outgoing channel, except for possibly the channels leading to the origin and the sender.

Proof: Assume the opposite. There exists an algorithm $\mathcal{A}$ that timely delivers message $m$ from the origin $x$ to all processes in the network such that some process $y$ receives it timely yet does not forward it to some process $z \neq x$.

Consider the propagation graph $T$ for $m$ to be as follows.

$$
x \rightarrow y \rightarrow z \rightarrow \text { rest of the processes }
$$

That is, the timely paths to all processes lead from $x$ to $y$ then to $z$. If $\mathcal{A}$ is such that $x$ sends $m$ to $y$, then, by assumption, $y$ does not forward $m$ to $z$. Therefore, no process except for $y$ gets $m$ through timely packets. By definition of the timely message, $m$ is not timely received by these processes. If $x$ does not send $m$ to $y$, then none of the processes receive a timely message. In either case, contrary to the initial assumption, $\mathcal{A}$ does not timely deliver $m$ to all processes in the network.

The below corollary follows from Lemma 1 .

Corollary 2. It requires $\Omega\left(n^{2}\right)$ packets to timely deliver a message in the general propagation model.

Combining Corollary 2 and Theorem 5 we obtain Corollary 3 , 
Corollary 3. In the general propagation model, there does not exist a message and packet efficient implementation of Omega.

Proposition 3. There exists a message efficient implementation of Omega in the general propagation model where each correct process can send reliable messages to the leader.

The algorithm that proves the above proposition is a straightforward extension of the second algorithm in Aguilera et al. [1] where every process re-sends received messages to every outgoing channel.

\section{Dependable channel model properties.}

Lemma 2. In any message efficient implementation of Omega, each correct process must have a fair-lossy path to the leader.

Proof: Assume there is a message-efficient implementation $\mathcal{A}$ of Omega where there is a correct process $x$ that does not have a fair-lossy path to the leader. According to Corollary 1, $x$ itself may not be elected the leader. Assume there is a computation $\sigma_{1}$ of $\mathcal{A}$ where process $y \neq x$ is elected the leader. Note that fair-lossy channels are not type discriminating. That is, if $x$ does not have a fair-lossy path to $y$, but has a fair lossy path to some other process $z$, then $z$ does not have a fair-lossy path to $y$ either. Thus, there must be a set of processes $S \subset N$ such that $x \in S$ and $y \notin S$ that do not have fair-lossy paths to processes outside $S$.

Since $\mathcal{A}$ is message efficient, processes of $S$ only send a finite number of messages to $y$. Consider another computation $\sigma_{2}$ which shares prefix with $\sigma_{2}$ up to the point were the last message from processes of $S$ is received outside of $S$. After that, all messages from $y$ to processes in $S$ and all messages from $S$ to outside are lost. That is in $\sigma_{2}, y$ does not have timely, or every fair-lossy, paths to processes of $S$. It is possible that some other process $w$ is capable of timely communication to all processes in the network. However, since $\mathcal{A}$ is efficient, no other processes but $y$ is supposed to send infinitely many messages.

Since all messages from $S$ are lost, $\sigma_{1}$ and $\sigma_{2}$ are indistinguishable for the correct processes outside $S$. Therefore, they elect $y$ as the leader. However, processes in $S$ receive no messages from $y$. Therefore, they have to elect some other process $u$ to be the leader. This means that $\mathcal{A}$ allows correct processes to output different leaders. That is, $\mathcal{A}$ is not an implementation of Omega.

We define a source to be a process that does not have incoming timely channels.

Lemma 3. To timely deliver a message in the dependable channel model, each recipient needs to send it across every outgoing channel to a source, except for possibly the channels leading to the origin and the sender.

The proof of the above lemma is similar to the proof of Lemma 1 . Observe that Lemma 3 states that the timely delivery of a packet requires $n$ messages per source. If the number of sources is proportional to the number of processes in the network, we obtain the following corollary. 
Corollary 4. It requires $\Omega\left(n^{2}\right)$ packets to timely deliver a message in the dependable channel model where the number of sources is proportional to $n$.

Theorem 6. In the dependable channel model, the following conditions are necessary and sufficient for the existence of a packet and message efficient implementation of Omega: (i) there is at least one process $l$ that has an eventually timely path to every correct process (ii) every correct process has a fair-lossy path to $l$.

Proof: We demonstrate sufficiency by presenting, in the next section, an algorithm that implements Omega in the dependable channel model with the conditions of the theorem.

We now focus on proving necessity. Let us address the first condition of the theorem. Assume there is a message and packet efficient implementation $\mathcal{A}$ of Omega in the dependable channel model even though no process has eventually timely paths to every correct process. Let there be a computation of $\mathcal{A}$ where some process $x$ is elected the leader even though $x$ does not have a timely path to each correct process. According to Corollary 1, $x$ needs to send infinitely many timely messages. According to Corollary 4 , each such message requires $\Omega\left(n^{2}\right)$ packets. That is, $\mathcal{A}$ may not be message and packet efficient. This proves the first condition of the theorem. The second condition immediately follows from Lemma 2 .

The below theorem shows that (plain) efficiency is all that can be achieved with the necessary conditions of Theorem 6. That is, even if these conditions are satisfied, supper packet efficiency is not possible.

Theorem 7. There does not exist a message and super packet efficient implementation of Omega in the dependable communication model even if there is a process $l$ with an eventually timely path to every correct process and every correct process has a fair-lossy path to $l$.

Proof: Assume the opposite. Suppose there exists a super packet efficient algorithm $\mathcal{A}$ that implements Omega in the network where some process $l$ has an eventually timely path to all correct processes and every correct process has fair-lossy paths to $l$.

Without loss of generality, assume the number of processes in the network is even. Divide the processes into two sets $S_{1}$ and $S_{2}$ such that the cardinality of both sets is $n / 2$. Refer to Figure 1 for illustration. $S_{1}$ is completely connected by timely channels. Similarly, $S_{2}$ is also completely connected by timely channels. The dependability of channels between $S_{1}$ and $S_{2}$ is immaterial at this point.

Consider a computation $\sigma_{1}$ of $\mathcal{A}$ on this network where all processes in $S_{1}$ are correct and all processes in $S_{2}$ crashed in the beginning of the computation. Since $\mathcal{A}$ is an implementation of Omega, one process $l_{1} \in S_{1}$ is elected the leader. Since $\mathcal{A}$ is message efficient, only $l_{1}$ sends messages infinitely often. Since $\mathcal{A}$ is super packet efficient, only $O(n)$ channels carry theses messages infinitely often. Since the network is completely connected, there are $(n / 2)^{2}$ channels leading from $S_{1}$ to $S_{2}$. This is in $O\left(n^{2}\right)$. Thus, there is least one channel $(x, y)$ such that $x \in S_{1}$ and $y \in S_{2}$ that does not carry messages from $l_{1}$ infinitely often. 


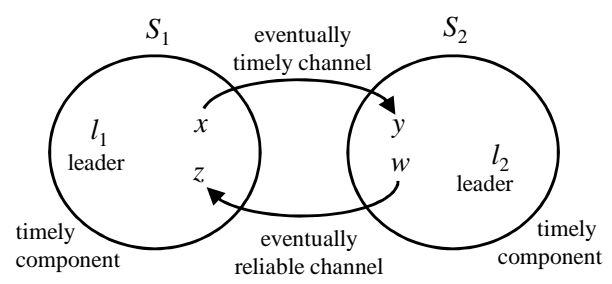

Fig. 1. Network for $\sigma_{3}$ computation of Theorem 7 .

Let us consider a computation $\sigma_{2}$ of $\mathcal{A}$ where all processes $S_{2}$ are correct and all processes in $S_{1}$ crash in the beginning of the computation. Similar to $\sigma_{1}$, there is a process $l_{2} \in S_{2}$ that is elected the leader in $\sigma_{2}$, and there is a channel $(z, w)$ such that $z \in S_{2}$ and $w \in S_{1}$ that carries only finitely many messages of $l_{2}$.

We construct a computation $\sigma_{3}$ of $\mathcal{A}$ as follows. All processes are correct. Channel dependability inside $S_{1}$ and $S_{2}$ is as described above. All channels between $S_{1}$ and $S_{2}$ are completely lossy, i.e., they lose every transmitted message. An exception is channel $(x, y)$ that becomes timely as soon as it loses the last message it is supposed to transmit. Similarly, channel $(z, w)$ becomes reliable as soon as it loses the last message.

To construct $\sigma_{3}$, we interleave the actions of $\sigma_{1}$ and $\sigma_{2}$ in an arbitrary manner. Observe that to processes in $S_{1}$ computations $\sigma_{1}$ and $\sigma_{3}$ are indistinguishable. Similarly, to processes in $S_{2}$, the computations $\sigma_{2}$ and $\sigma_{3}$ are indistinguishable.

Let us examine the constructed computation closely. Sets $S_{1}$ and $S_{2}$ are completely connected by timely channels, and $(x, y)$, connecting $S_{1}$ and $S_{2}$ is eventually timely. This means that $l_{1}$ has an eventually timely path to every correct process in the network. Moreover, due to channel $(z, w)$, every process has a fair-lossy path to $l_{1}$. That is, the conditions of the theorem are satisfied. However, the processes of $S_{1}$ elect $l_{1}$ as their leader while the processes of $S_{2}$ elect $l_{2}$. This means that the processes do not agree on the single leader. That is, contrary to the initial assumption, $\mathcal{A}$ is not an implementation of Omega. The theorem follows.

\section{$5 \mathcal{M P O}$ : Message and Packet Efficient Implementation of Omega}

In this section we present an algorithm we call $\mathcal{M P O}$ that implements Omega in the fairlossy channel communication model. As per Theorem 6, we assume that there is at least one process that has an eventually timely path to every correct process in the network and every correct process has a fair-lossy path to this process.

Algorithm outline. The code of the algorithm is shown in Figure 2, The main idea of $\mathcal{M P O}$ is for processes to attempt to claim the leadership of the network while discovering the reliability of its channels. Each process weighs each channel by the number of messages that fail to come across it. The lighter channel is considered more reliable. If a process determines that it has the lightest paths to all processes in the network, the process tries to claim leadership of the network. 
constants

$p / /$ process identifier

$N / /$ set of network process identifiers, cardinality is $n$

timers $[p]$ length is $T O$

variables

leader, initially $\perp / /$ local leader

phases $[n]$, initially zero // current phase number

edges $[n][n]$, initially zero // edge fault weights

$\operatorname{arbs}[n]$, initially arbitrary // arborescences

timer $s[n]$, initially timers $[p]$ on, others off

length of timers $[x]: x \neq p$ is arbitrary // timer to send/receive a message

shout, initially zero // process id to send alive to all neighbors

actions

timeout $($ timers $[q]) \longrightarrow$

if $p=q$ then $/ /$ own/sender timeout, compute arb rooted in $p$ based on edges

newArb $=$ arborescence $($ edges,$p)$

newLeader $:=\operatorname{minWeight}((\operatorname{arbs}[r]: r \neq p:$ on $($ timer $s[r]))$, newArb $))$

if leader $\neq$ newLeader then // leadership changes

if newLeader $=p$ then $/ / p$ gains leadership

$\operatorname{arbs}[p]:=$ newArb

send $\operatorname{startPhase}(p$, phases $[p], \operatorname{arbs}[p])$ to $N / p$

if leader $=p$ then $/ / p$ loses leadership

phases $[p]:=$ phases $[p]+1$

send stopPhase (p,phases $[p])$ to $N / p$

leader $:=$ newLeader

else // leadership persists

if leader $=p$ then

shout $:=$ shout $+1 \bmod n$

if shout $\neq p$ then

send alive( $p$, phases $[p]$, shout) to arbs $[p]$ (p.children)

else // my turn to shout

send alive ( $p$, phases $[p]$, shout) to $N / p$

reset (timer $s[p]) / /$ own timer never off

else // neighbor timeout/receiver timeout, assume failed, increase, do not reset send failed ( $q, p, \operatorname{arbs}[q](p$.parent $))$ to $N / p$

increase (timers $[q]$ )

receive startPhase ( $q$, phase, arb) for the first time $\longrightarrow$

// if new phase, propagate message, reset timer

if $p \neq q \wedge$ phase $[q] \leq$ phase then

$\operatorname{arbs}[q]:=\operatorname{arb}$

phase $[q]:=$ phase

send startPhase (q, phase, arb) to $N / p$

reset(timers $[q])$

receive stopPhase (q, phase) for the first time $\longrightarrow$

if $p \neq q \wedge$ phase $[q]<$ phase then

phases $[q]:=$ phase

send stopPhase (q, phase) to $N / p$

stop $($ timers $[q])$

receive alive ( $q$, phase, $s h$ ) for the first time from $r \longrightarrow$

if $p \neq q \wedge$ phase $[q]=$ phase then

if $r=\operatorname{arbs}[q]($ p.parent $)$ then $/ /$ received through arborescence

if $s h \neq p$ then

send alive $(q$, phase, sh) to $\operatorname{arbs}[q]$ (p.children)

else // my turn to shout

send alive (q,phase, sh) to $N / p$

reset(timers $[q])$

else // received from elsewhere

if off (timers $[q])$ then

reset(timers $[q]$ )

receive failed $(q, r, s)$ for the first time $\longrightarrow$

if $p=q$ then $/ /$ if $p$ 's alive failed

edges $[s][r]:=$ edges $[s][r]+1 / /$ increase weight of edge from parent

else

send failed $(q, r, s)$ to $N / p$

Fig. 2. Message and packet efficient implementation of Omega $\mathcal{M P O}$. 
The leadership is obtained in phases. First, the leader candidate sends startPhase message. Then, the candidate periodically sends alive message. In case an alive fails to reach one of the processes on time, the recipient replies with failed. The size of startPhase depends on the network size. The size of the other message types is constant.

The routes of the messages vary. Messages that are only sent finitely many times are broadcast: sent across every channel in the network. Once one process receives such a message for the first time, the process re-sends it along all of its outgoing channels. Specifically, startPhase, stopPhase and failed are broadcast. The leader sends alive infinitely often. Hence, for the algorithm to be packet efficient, alive has to be sent only along selected channels. Message alive is routed through the channels that the origin believes to be the most reliable.

Specifically, alive is routed along the channels of a minimum weight arborescence: a directed tree rooted in the origin reaching all other processes. The arborescence is computed by the origin once it claims leadership. It is sent in the startPhase that starts a phase. Once each process receives the arborescence, the process stores it in the arbs array element for the corresponding origin. After receiving alive from a particular origin, the recipient consults the respective arborescence and forwards the message to the channels stated there.

In addition to routing alive along the arborescence, each process takes turns sending the leader's alive to all its neighbors. The reason for this is rather subtle: see Theorem 7 for details. Due to crashes and message losses, arbs for the leader at various processes may not reach every correct process. For example, it may lead to a crashed process. Thus, some processes may potentially not receive alive and, therefore, not send failed. Since failed are not sent, the leader may not be able to distinguish such a state from a state with correct arbs.

To ensure that every process receives alive, each process, in turn, sends alive to its every neighbor rather than along most reliable channels. Since only a single process sends to all neighbors a particular alive message, the packet complexity remains $O(n)$.

Message failed is sent if a process does not receive a timely alive. This message carries the parent of the process which was supposed to send the alive. That is, the sender of failed blames the immediate ancestor in the arborescence. Once the origin of the missing alive, receives failed, it increments the weight of the appropriate edge in edges that stores the weights of all channels. If a process has timely outgoing paths to all processes in the network, its arborescence in edges convergences to these paths.

Action specifics. The algorithm is organized in five actions. The first is a timeout action, the other four are message-receipt actions.

The timeout action handles two types of timers: sender and receiver. Process $p$ 's own timer $(q=p)$ is a sender timer. It is rather involved. This timer is always on since the process resets it after processing. First, the process computes the minimum weight of the arborescence for each leader candidate. A process is considered a leader candidate if its timer is on. Note that since $p$ 's own timer is always on, it is always considered.

The process with the minimum weight arborescence is the new leader. If the leadership changes (leader $\neq$ newLeader), further selection is made. If $p$ gains leadership 
(newLeader $=p$ ), then $p$ starts a new phase by updating its own minimum-weight arborescence and broadcasting startPhase. If $p$ loses leadership, it increments its phase and broadcasts stopPhase bearing the new phase number.

If the leadership persists (leader $=$ newLeader) and $p$ is the leader, it sends alive. Process $p$ keeps track of whose turn it is to send alive to all its neighbors in the shout variable. The variable's value rotates among the ids of all processes in the network.

The neighbor timer $(q \neq p)$ is a receiver timer. If the process does not get alive on time from $q$, then $p$ sends failed. In case the process sends failed, it also increases the timeout value for the timer of $q$ thus attempting to estimate the channel delay.

For our algorithm, the timer integers are as follows. The sender timer is an arbitrary constant integer value $T O$. This value controls how often alive is sent. It does not affect the correctness of the algorithm. Receiver timers initially hold an arbitrary value. The timer integer is increased every time there is a timeout. Thus, for an eventually timely channel, the process is able to estimate the propagation delay and set the timer integer large enough that the timeout does not occur. For untimely channels, the timeout value may increase without bound.

The next four actions are message receipt handling. Note that a single process may receive packets carrying the same message multiple times across different paths. However, every process handles the message at most once: when it encounters it for the first time. Later duplicate packets are discarded.

The second action is startPhase handling. The process copies the arborescence and phase carried by the message, rebroadcasts it and then resets the alive receiver timer associated with the origin process. The third action is the receipt of stopPhase which causes the recipient to stop the appropriate timer.

The forth action is alive handling. If alive is the matching phase, it is further considered. If alive comes through the origin's arborescence, the receiver sends alive to its children in the origin's arborescence or broadcasts it. The process then resets the timer to wait for the next alive. If alive comes from elsewhere, that is, it was the sender's turn to send alive to all its neighbors, then $p$ just resets the timeout and waits for an alive to arrive from the proper channel. This forces the process to send failed if alive does not arrive from the channel of the arborescence.

The last action is failed handling. If failed is in response to an alive originated by this process $(p=q)$ then the origin process increments the weight of the edge from the parent of the reporting process to the process itself according to the message arborescence. If failed is not destined to this process, $p$ rebroadcasts it.

\section{$6 \mathcal{M P O}$ Correctness Proof}

Correctness proof definitions. Throughout this section, $l$ is the identifier of the process that has eventually timely paths to all other processes. For simplicity, we assume that $l$ is the single such process. Denote $B$ as the maximum number of steps in any timely channel propagation delay. Process $p$ is a local leader if leader $_{p}=p$, i.e., the process elected itself the leader. A process may be a local leader but not the global leader. That is, several processes may be local leaders in the same state. Let realArbs $(x)$ for the origin process $x$ be 
the relation defined by $\operatorname{arbs}[x]$ (y.children) at every process $y$. That is, $\operatorname{realArbs}(x)$ is the distributed relation that determines how alive messages are routed if they are originated by $x$.

Lemma 4. For any local leader process $x$ and another correct process y such that $y$ is not reachable from $x$ through timely channels over correct processes in realArbs $(x)$, either $(i)$ realArbs $(x)$ changes or (ii) $x$ loses leadership, changes phase or receives infinitely many failed messages.

Proof: To prove the lemma, it is sufficient to show that if realArbs $(x)$ does not change and $x$ does not lose the leadership or change phase, then $x$ receives infinitely many failed.

Let $S$ be a set of correct processes that are reachable from $x$ through timely channels and through correct processes in realArbs $(x)$. Since $y$ is not reachable from $x, S \neq N$. Recall that every process has fair-lossy paths to all processes in the network. Therefore, there is such a path from $x$ to $y$. This means that there is a process $z \in S$ such that it has a fair-lossy channel to $w \notin S$.

Let us examine process $w$ closer. The network is completely connected. Therefore, all other processes from $S$ have channels to $w$. Note that at least one channel, from $z$ is fair-lossy. Moreover, since $w$ does not belong to $S$, if $\operatorname{realArbs}(x)$ reaches $w$, the path to $w$ is not timely.

Since $x$ is a local leader and does not lose its leadership, it sends infinitely many alive messages. Other processes forward these alive along realArbs $(x)$. Also, by the design of the algorithm, every process takes turn sending alive to all of its neighbors rather than forwarding it along realArbs $(x)$. Let us examine the receipt of these messages by $w$.

Process $z$ belongs to $S$. That is, the path from $x$ to $z$ in $\operatorname{realArbs}(x)$ is timely. This means that it receives and sends infinitely many alive originated by $x$. Since the channel from $z$ to $w$ is fair-lossy, infinitely many of these alive are delivered to $w$. In addition, $w$ possibly receives alive from other processes of $S$. Since, none of these channels are part of realArbs $(x)$, when $w$ receives alive from processes in $S$, it resets the corresponding receive timer only when the timer is off. The timer is turned off only when the timeout is executed and failed is broadcast.

The only possible way this receive timer is reset without the timeout action execution is when $w$ receives alive through realArbs $(x)$. However, the path from $x$ to $w$ in realArbs $(x)$ is not timely. By the definition of non-timely paths, there are infinitely many computation segments of arbitrary fixed length where no alive from $x$ is delivered to $w$. This means that, regardless of the timeout variable value at $w$, the alive messages generate receiver timeouts. That is, infinitely many timeouts are executed at $w$. Each timeout generates a failed message broadcast by $w$. Since there are infinitely many broadcasts, infinitely many succeed in reaching $x$. Hence, the lemma.

Lemma 5. If each process $x \neq l$ is a local leader in infinitely many states then it receives infinitely many failed messages.

Proof: Let $x \neq l$ be a local leader in infinitely many states of a particular computation of the algorithm. Once a process assumes local leadership, it may lose it either by (i) in- 
creasing the weight of its minimum weight arborescence (ii) by recording an arborescence $\operatorname{arbs}[y]$ for a process $y$ with lower weight than $\operatorname{arbs}[x]$.

A process increases the weight of its arborescence only when it gets a failed message. Thus, to prove the lemma we need to consider the second case only.

Since $x$ is a local leader in infinitely many states, it must gain local leadership back after losing it to another process $y$. By the design of the algorithm, the weight of the arborescence of any process in arbs may only increase. This means that once $x$ gains the leadership back from $y, x$ may not lose it to $y$ again without increasing the weight of its own minimum weight arborescence. Thus, either $x$ increases the weight of its arborescence or, eventually, it has the lightest arborescence among the leader candidates.

In case $x$ has the lightest arborescence, it either becomes heavier than some other leader candidate's or $x$ gets infinitely many failed. However, only the latter part of the statement needs to be proven since $x$ gains leadership infinitely often.

If $x$ is a local leader, it does not send startPhase or stopPhase. Let us consider the state where all startPhase packets are delivered. In this case realArbs $(x)$ does not change. Since $x \neq l$, even if all correct processes are reachable from $x$ in realArbs $(x)$, some links in $\operatorname{realArbs}(x)$ are not timely. Then, according to Lemma 4, $x$ gets infinitely many failed.

To summarize, if $x \neq l$ is a local leader in infinitely many states, it receives infinitely many failed.

Lemma 6. Process $l$ is a local leader in infinitely many states.

Proof: According to Lemma 5 either each process $x \neq l$ stops gaining local leadership or the weight of its minimum arborescence grows infinitely high. If the latter is the case, $x$ has to gain and lose local leadership infinitely often. In this case, it sends startPhase infinitely may times. Message startPhase is broadcast. Since every process $x$ has fairlossy paths to $l$, by the definition of fair-lossy paths, infinitely many broadcasts succeed. This means that the weight of $\operatorname{arbs}[x]$ at $l$ grows without bound. Therefore, if $l$ loses local leadership, it gains it back infinitely often.

The below lemma follows immediately from the operation of the algorithm.

Lemma 7. The timer length of timers[l] at every process either stops increasing or it reaches $T O+B *(n-1)$

And the below lemma follows from the assumption that the leader has an eventually timely path to every correct process.

Lemma 8. In every computation, there is a suffix where each broadcast message sent by l is timely delivered to every correct process.

Lemma 9. An edge leading to process $x$ in a timely path in realArbs $(l)$ at $l$ generates only finitely many failed.

Proof: The origin starts every phase with startPhase, then periodically sends zero or more alive and then possibly ends the phase with a stopPhase that carries the phase number greater than alive and startPhase. 
Message failed is generated only when the timer expires at the receiving process. The timer is reset by startPhase and alive. The timer is stopped by stopPhase.

We prove the lemma by showing that the timer reset by messages of a particular phase expires only finitely many times. We start our consideration from the point of the computation where the conditions of Lemmas 7 and 8 hold.

Only alive and startPhase may reset the timeout. Since the conditions of Lemma 8 hold, startPhase is delivered within $B(n-1)$ computation steps to all processes. Message alive may be received earlier than startPhase. However, since such alive carries a phase number that differs from the number stored at the recipient process, the message is ignored. If alive arrives after startPhase, the reasoning is similar to the case where alive is sent after startPhase which is to be considered next.

Every alive sent after startPhase delivery, travels over the timely path in realArbs $(l)$. At most every $T O$ number of steps, either another alive or stopPhase is sent. Since the path in realArbs $(l)$ is timely, alive arrives at most after $T O+B(n-1)$ steps. Due to Lemma 8, the same is true of stopPhase. That is, after alive is received, either another alive or stopPhase is received within $T O+B(n-1)$ steps. The receipt of alive resets the timeout. The receipt of stopPhase stops it. Due to Lemma 7, the timer does not expire.

Moreover, after the receipt of stopPhase, the subsequent alive messages are ignored since stopPhase carries a greater phase number. That is, after stopPhase is received, the timer is never reset or expires due to the messages of this phase.

Lemma 10. Every untimely edge in realArbs(l) leading to a correct process either gets removed or l gets infinitely many failed messages.

Proof: Due to Lemma 6, process $l$ is a local leader in infinitely many states. Through the argument similar to that of Lemma 5, we can show that eventually either $l$ gets failed and increases the weight of its minimum arborescence or its minimum arborescence becomes the lightest among the leader candidates. Then, $l$ can lose leadership only if it gets failed.

In this case, according to Lemma 4, $l$ receives infinitely many failed messages or either loses leadership, changes phase or changes realArbs $(l)$. Observe that $l$ may change phase only when it receives failed. It loses leadership only if it gets failed. The change of realArbs $(l)$ happens only when $l$ broadcasts startPhase after changing phase and, therefore, getting failed. Due to Lemma 6, it gains the leadership back infinitely often.

That is, in any case, as long as $\operatorname{realArbs}(l)$ contains an untimely edge leading to a correct process, $l$ gets infinitely many failed.

The below lemma follows from Lemmas 9 and 10 .

Lemma 11. Every computation of $\mathcal{M P O}$ contains a suffix where each channel of realArbs $(l)$ is timely.

Lemma 12. Every computation of $\mathcal{M P O}$ contains a suffix where realArbs $(l)$ is the same as arbs[l] in process $l$. 
Proof: We start our consideration from the point where the conditions of Lemma 11 hold. Suppose realArbs $(l)$ and $\operatorname{arbs}[l]$ differ for some process $x$. By the design of the algorithm, this may happen only if $\operatorname{arbs}[l]$ in process $x$ has an earlier phase than in $l$. However, since phases differ, alive sent by $l$ are ignored by $x$. This leads to either $x$ sending fail to $l$ or claiming leadership. In either case, $l$ sends startPhase. According to Lemma 8, this broadcasts succeeds which synchronizes $\operatorname{arbs}[l]$ and $\operatorname{realArbs}(l)$.

Theorem 8. Algorithm $\mathcal{M P O}$ is a message and packet efficient implementation of Omega in the fair-lossy channel model.

Proof: $\quad$ First, we prove that $\mathcal{M P O}$ implements Omega. Indeed, lemma 6 shows that $l$ is a local leader in infinitely many states. Lemmas 9 and 10 show that $l$ gets finitely many failed. According to Lemma 5, every process $x \neq l$ either stops being a local leader or gets infinitely many failed. This means that at any process the arborescence of $l$ will eventually be lighter than any other leader contender.

According to Lemma 6, $l$ sends infinitely many alive messages along realArbs $(l)$. Due to Lemma 10, realArbs $(l)$ eventually has no untimely channels. Since $l$, according to Lemma 10 , receives only finitely many failed, due to Lemma 4 , realArbs $(l)$ eventually has timely paths from $l$ to every correct process. According to Lemma 12, realArbs $(l)$ and $\operatorname{arbs}[l]$ are eventually the same.

This means that $l$ will be a leader contender in every correct process. Since it has the lightest arborescence, it becomes the leader at every correct process. In other words, $\mathcal{M P O}$ is a correct implementation of Omega.

By the design of the algorithm, once $l$ has the lightest arborescence and all correct processes drop out of leadership contention, $l$ is the only process that sends alive messages. By definition, $\mathcal{M P O}$ is message efficient.

The messages are routed along $\operatorname{arbs}[l]$. It is an arborescence. Hence, the number of such messages is in $O(n)$. In addition, each process takes a turn sending alive to its neighbors. This is another $O(n)$ packets. Therefore, the packet complexity of $\mathcal{M P O}$ is in $O(n)$.

\section{Algorithm Extensions}

We conclude the paper with several observations about $\mathcal{M P O}$. The algorithm trivially works in a non-completely connected network provided that the rest of the assumptions used in the algorithm design, such as eventually timely paths from the leader to all correct processes, are satisfied. Similarly, the algorithm works correctly if the channel reliability and timeliness is origin-related. That is, a channel may be timely for some, not necessarily incident, process $x$, but not for another process $y$.

Algorithm $\mathcal{M P O}$ may be modified to use only constant-size messages. The only nonconstant size message is startPhase. However, the message type is supposed to be timely. So, instead of sending a single large message, the modified $\mathcal{M P O}$ may instead send a sequence of fixed-size messages with the content to be re-assembled by the receivers. If one of the constituent messages does not arrive on time, the whole large message is considered lost. 


\section{References}

1. Marcos Kawazoe Aguilera, Carole Delporte-Gallet, Hugues Fauconnier, and Sam Toueg. On implementing omega in systems with weak reliability and synchrony assumptions. Distributed Computing, 21(4):285-314, 2008.

2. Antonio Fernández Anta and Michel Raynal. From an asynchronous intermittent rotating star to an eventual leader. IEEE Trans. Parallel Distrib. Syst., 21(9):1290-1303, 2010.

3. Martin Biely and Josef Widder. Optimal message-driven implementations of omega with mute processes. $A C M$ Transactions on Autonomous and Adaptive Systems (TAAS), 4(1):4, 2009.

4. Béla Bollobás. Random graphs. Cambridge University Press, 2 edition, October 2001.

5. Tushar Deepak Chandra, Vassos Hadzilacos, and Sam Toueg. The weakest failure detector for solving consensus. Journal of ACM, 43(4):685-722, 1996.

6. Tushar Deepak Chandra and Sam Toueg. Unreliable failure detectors for reliable distributed systems. Journal of the ACM, 43(2):225-267, 1996.

7. Bernadette Charron-Bost, Matthias Függer, and Thomas Nowak. Approximate consensus in highly dynamic networks. arXiv preprint arXiv:1408.0620 2014.

8. Carole Delporte-Gallet, Stéphane Devismes, Hugues Fauconnier, and Mikel Larrea. Algorithms for extracting timeliness graphs. In Structural Information and Communication Complexity, 17th International Colloquium, SIROCCO 2010, Sirince, Turkey, June 7-11, 2010. Proceedings, pages 127-141, 2010.

9. Michael J. Fischer, Nancy A. Lynch, and Michael S. Paterson. Impossibility of distributed consensus with one faulty process. J. $A C M, 32(2): 374-382,1985$.

10. E. N. Gilbert. Random graphs. Ann. Math. Statist., 30(4):1141-1144, 121959.

11. Martin Hutle, Dahlia Malkhi, Ulrich Schmid, and Lidong Zhou. Chasing the weakest system model for implementing $\omega$ and consensus. IEEE Trans. Dependable Sec. Comput., 6(4):269-281, 2009.

12. Alberto Lafuente, Mikel Larrea, Iratxe Soraluze, and Roberto Cortiñas. Communication-optimal eventually perfect failure detection in partially synchronous systems. Journal of Computer and System Sciences, 81(2):383-397, 2015.

13. Mikel Larrea, Antonio Fernández, and Sergio Arévalo. On the implementation of unreliable failure detectors in partially synchronous systems. IEEE Trans. Computers, 53(7):815-828, 2004.

14. Dahlia Malkhi, Florin Oprea, and Lidong Zhou. $\omega$ meets paxos: Leader election and stability without eventual timely links. In Distributed Computing, pages 199-213. Springer, 2005.

15. Achour Mostefaoui, Eric Mourgaya, and Michel Raynal. Asynchronous implementation of failure detectors. In 2013 43rd Annual IEEE/IFIP International Conference on Dependable Systems and Networks (DSN), pages 351351. IEEE Computer Society, 2003.

16. Achour Mostéfaoui, Michel Raynal, and Corentin Travers. Time-free and timer-based assumptions can be combined to obtain eventual leadership. IEEE Trans. Parallel Distrib. Syst., 17(7):656-666, 2006.

17. Roberto De Prisco, Butler W. Lampson, and Nancy A. Lynch. Revisiting the PAXOS algorithm. Theor. Comput. Sci., 243(1-2):35-91, 2000. 